Check for updates

Cite this: Mater. Chem. Front. 2020, 4, 1507

Received 14th January 2020, Accepted 25th March 2020

DOI: $10.1039 / \mathrm{d} 0 \mathrm{qm} 00016 \mathrm{~g}$

rsc.li/frontiers-materials

\section{The role of dipole moment in two fused-ring electron acceptor and one polymer donor based ternary organic solar cells $\dagger$}

\author{
Xunfan Liao, $\ddagger^{\mathrm{ab}}$ Yongjie Cui, $\ddagger^{\mathrm{b}}$ Xueliang Shi, (D)*c Zhaoyang Yao, ${ }^{\mathrm{d}}$ Heng Zhao, \\ Yongkang An, ${ }^{f}$ Peipei Zhu, ${ }^{a}$ Yaxiao Guo, ${ }^{d}$ Xiang Fei, ${ }^{b}$ Lijian Zuo, (D) ${ }^{g}$ Ke Gao, (D) 9 \\ Francis Lin, ${ }^{g}$ Qian Xie, ${ }^{f}$ Lie Chen, (D) ${ }^{f}$ Wei Ma, (ID *e Yiwang Chen (D) *a and \\ Alex K.-Y. Jen*gh
}

\begin{abstract}
Fused-ring electron acceptor (FREA) based ternary organic solar cells (OSCs) have made significant progress and attracted considerable attention due to their simple device architecture and broad absorption range in devices. There are three key parameters that need to be fine-tuned in ternary OSCS including absorption, energy level and morphology in order to realize high efficiencies. Herein, a series of FREAs with diverse electron-rich cores or electron-deficient terminals are developed and rationally combined to achieve high performance ternary OSCs. The dipole moment of FREAs' terminals has been unveiled as an important factor and its working mechanism has been thoroughly investigated by systematically studying six ternary OSCs. These ternary blends all exhibit complementary absorption and cascade energy levels, which can facilitate efficient light-harvesting and charge transfer. Additionally, the morphological effects on ternary OSCs are eliminated through comparative studies while demonstrating distinctively different performance. The preliminary results show that compatible dipole moment between two FREAs is critical in ternary blends. Specifically, the performance of the ternary system with two FREAs having quite different dipole moment terminals is worse compared to that with similar terminal dipole moments. The pair with larger difference in the dipole moment will also negatively impact device performance. This interesting phenomenon is likely due to the fact that very different dipole moments of terminals in FREAs can significantly decrease the electron mobility as well as induce unbalanced hole/electron transport. Consequently, it results in increased charge recombination and reduced charge collection efficiency. This finding demonstrates that the dipole moment of FREAs should be taken into account in designing ternary OSCs.
\end{abstract}

${ }^{a}$ Institute of Advanced Scientific Research, Jiangxi Normal University, 99 Ziyang Avenue, Nanchang 330022, China. E-mail: ywchen@ncu.edu.cn

${ }^{b}$ State Key Laboratory for Modification of Chemical Fibers and Polymer Materials \& College of Materials Science and Engineering, Donghua University, Shanghai 201620, China

'Shanghai Key Laboratory of Green Chemistry and Chemical Processes, School of Chemistry and Molecular Engineering, East China Normal University, Shanghai 200062, China. E-mail: xlshi@chem.ecnu.edu.cn

${ }^{d}$ Department of Chemistry, Organic/Applied Physical Chemistry, KTH Royal Institute of Technology, Stockholm 10044, Sweden

${ }^{e}$ State Key Laboratory for Mechanical Behavior of Materials, Xi'an Jiaotong University, Xi'an 710049, China. E-mail: msewma@xjtu.edu.cn

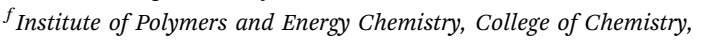

Nanchang University, Nanchang 330031, China

${ }^{g}$ Department of Materials Science and Engineering, University of Washington, Seattle,WA 98195, USA. E-mail: ajen@uw.edu

${ }^{h}$ Department of Chemistry, City University of Hong Kong, Kowloon, Hong Kong

$\dagger$ Electronic supplementary information (ESI) available. See DOI: 10.1039/d0qm00016g

\$ These authors contributed equally to this work.

\section{Introduction}

Non-fullerene acceptor (NFA) based organic solar cells (OSCs) have attracted great attention, and significant progress has been made due to their broad absorption in both visible and nearinfrared (NIR) regions, easily adjustable energy levels, and tunable crystallinity in active layer morphology. ${ }^{1-8}$ To achieve efficient OSCs, effective absorption of sunlight from the active layer is very critical. Thus, considerable efforts have been devoted to the design of active layer materials and device structures to ensure broadened absorption. ${ }^{9-19}$ For example, tandem solar cells by stacking multiple photoactive layers with complementary absorption spectra can capture more photon flux to improve the shortcircuit current density $\left(J_{\mathrm{sc}}\right)^{20-24}$ Although a high power conversion efficiency (PCE) of $17.3 \%$ was obtained, ${ }^{25}$ the application of tandem OSCs is still quite limited because of the complexity in device fabrication and large-scale production. 
Compared with tandem OSCs, ternary solar cells where the active layer typically consists of either two donors and one acceptor or one donor and two acceptors have the simplicity of the single junction structure and broadened absorption of the active layer. ${ }^{26-30}$ However, there are three key parameters including absorption, energy level and morphology that need to be fine-tuned simultaneously in order to realize efficient device performance. Although the absorption and energy level of the third component can be easily determined by photophysical and electrochemical measurements for the ternary system, the morphology of the ternary system is more difficult to control. ${ }^{31-33}$ Recently, Yan et al. reported an effective method of minimizing interfacial tension between two fused-ring electron acceptors (FREAs) to control the morphology ${ }^{34}$ of the ternary system. ${ }^{35-40}$ Although the PCE of this type of ternary OSCs has been significantly increased to over $16 \%,{ }^{41-44}$ a tedious trial-and-error approach involving the fabrication of a large number of devices is often employed. Therefore, it would be highly desirable to develop a simple and intuitive method to effectively predict what kind of FREA structures should be used in a ternary system.

It has been reported that charge separation and charge transfer processes involved in FREA based OSCs are very different from those of fullerene-based acceptors. ${ }^{45}$ For the blend based on a single polymer donor and a fullerene acceptor, the ultrafast charge transfer originates from the variation in the $\pi$-electron densities of the donor and the acceptor. However, it is very different for FREA based blends such as those using ITIC derivatives. These kinds of acceptors usually have electron-rich cores with terminal electron-deficient groups such as substituted 3-(dicyanomethylidene)-indan-1-one (IC) and its fluorinated or chlorinated derivatives. Considering the strong electron withdrawing di-cyano-substituted terminals in FREAs, there may be some dipole effect from the terminals that can facilitate the charge transfer and at the same time prevent bimolecular recombination when these terminals are near the electron-rich polymer donor. Besides, recent crystallographic analysis revealed that terminal ICs form intermolecular $\pi-\pi$ stacking with each other which might contribute to their efficient charge transfer. ${ }^{18}$ Therefore, the terminal's dipole moment or the electronegativity of FREAs may play a vital role in facilitating charge separation and transfer in the binary blends. ${ }^{46}$ Since there is no systematic investigation of these effects in FREA-based ternary OSCs, it is important to verify them and exploit the possibility of establishing a new material selection rule to expedite the development of this field.

In this work, we have developed a series of FREAs with different electron-rich cores or electron-deficient terminal groups and systematically studied them in six ternary blends to confirm the effect of 4TBA and 4TIC-4F, which have the largest difference in terminal dipole moment, in ternary OSCs. As shown in Scheme 1, we initially use the PBDT-2TC polymer

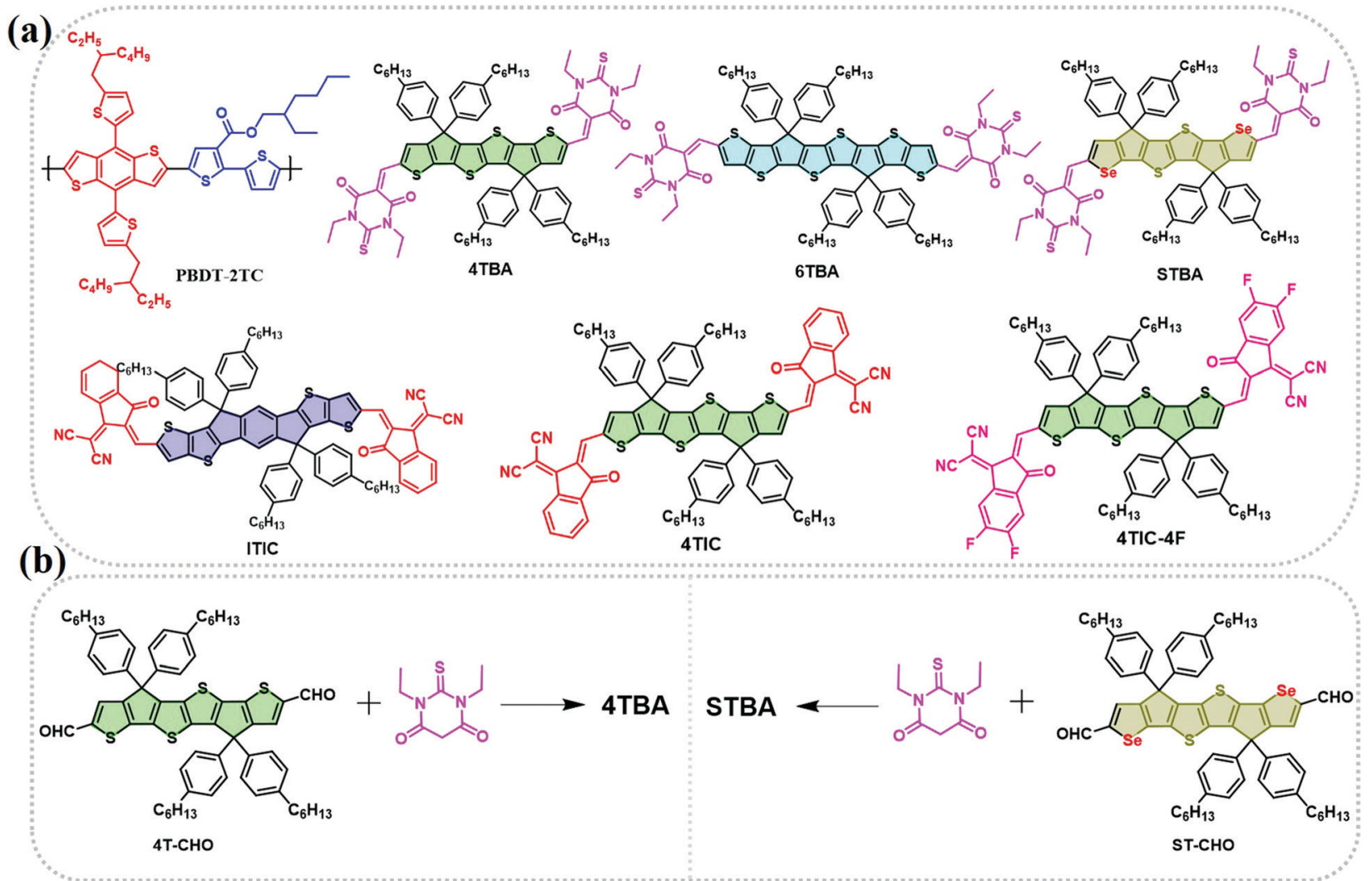

Scheme 1 (a) Molecular structures of the polymer donor PBDT-2TC and non-fullerene acceptors 4TBA, 6TBA, STBA, ITIC, 4TIC and 4TIC-4F. (b) The synthetic routes for 4TBA and STBA. 
synthesized in-house with suitable energy levels and intense absorption in the region between 400 and $650 \mathrm{~nm}$ as the donor to match with these FREAs. ${ }^{47}$ Two FREAs (4TBA and 6TBA) with a similar donor core and the same terminal 1,3-diethyl-2thioxodihydropyrimidine-4,6(1H,5H)-dione (BA) are combined with the PBDT-2TC polymer to form the first ternary blend PBDT2TC:4TBA:6TBA. Then, the second and third ternary blends PBDT-2TC:4TBA:ITIC and PBDT-2TC:4TBA:4TIC are formed by combining 4TBA with ITIC and 4TIC, respectively. These FREAs have a similar or the same donor core but with different terminal groups. The results show that ternary blends based on acceptors with different terminals have worse device performance than those with the same terminals; especially the PBDT-2TC:4TBA: 6TBA blend shows the best performance. The PBDT-2TC:4TBA:ITIC and PBDT-2TC:4TBA:4TIC devices have significantly lower electron mobility, which results in unbalanced hole/electron transport and increased charge recombination. These three ternary blends all exhibit complementary absorption and cascade energy levels, and the morphologies of these blend films are also quite similar. Therefore, there must be some other parameters that dictate the quite different performance of these ternary OSCs.

We then compare the terminals' dipole moment of these acceptors by DFT calculation. The results reveal that the dipole moment value for the different terminals, Th-BA, Th-IC and Th-DFIC, shows a gradual increase. To confirm our hypothesis, the acceptor $4 \mathrm{TIC}-4 \mathrm{~F}$ with the same donor core $4 \mathrm{~T}$ but with the largest dipole moment terminal 2-(5,6-difluoro-3-oxo-2,3-dihydro- $1 \mathrm{H}$ inden-1-ylidene)malononitrile (DFIC) was used to form the fourth ternary blend PBDT-2TC:4TBA:4TIC-4F. This blend only shows a little higher crystallinity than that of the binary blend while its electron mobility is the worst, leading to the most unbalanced uh/ue value and the poorest PCE. This result also reveals that larger dipole moment difference in FREAs will cause worse device performance.

To further verify the hypothesis and to exclude the interference from the donor core, another two ternary blends of PBDT-2TC:4TBA:STBA and PBDT-2TC:ITIC:4TIC with the same terminal but different donor cores were investigated as well. The novel acceptor, STBA, was synthesized by connecting a novel donor core, selenophene-thieno[3,2-b] thiophene-selenophene (ST), with the same terminal group, 4TBA. Although there is quite a huge difference in the donor core, the performance of the PBDT2TC:4TBA:STBA blend shows a small attenuation compared to that of the binary blend. The device of PBDT-2TC:ITIC:4TIC also works well with improved performance which can exclude the interference from the donor core. Therefore, the comparative studies of six ternary systems verify the importance of FREA dipole moment in choosing a suitable third component in ternary OSCs. Although this discovery may not be generally applicable to all systems, it does provide some guidance for further improving the performance of ternary OSCs.

\section{Results and discussion}

\subsection{Ternary blend design and quantum calculations}

The polymer donor employed in this work is PBDB-2TC (shown in Scheme 1), which has been previously reported by our group and showed good performance with FREAs. ${ }^{47}$ As shown in Scheme 1a, three terminals, BA, IC and DFIC, with different electronegativity are investigated, and their derived NIR FREAs such as 6 TBA, ${ }^{48} 4$ TIC, ${ }^{18}$ ITIC $^{1}$ and 4 TIC- $4 \mathrm{~F}^{49}$ are employed in this study. We have also developed another host acceptor 4TBA to evaluate the effects of different FREAs because it has a matched energy level and complementary absorption with polymer PBDT2TC and the above NIR FREAs. Four ternary blends PBDT-2TC: 4TBA:6TBA, PBDT-2TC:4TBA:ITIC, PBDT-2TC:4TBA:4TIC and PBDT-2TC:4TBA:4TIC-4F are carefully designed to study these ternary OSCs. In addition, a novel acceptor STBA with ST as the electron-rich core was also synthesized to systematically study the dipole moment effect in ternary OSCs by excluding the possible interference from the donor core.

4TBA and STBA were synthesized by the Knoevenagel condensation reaction (Scheme 1b) and the synthetic details and the related characterization are available in the ESI $\dagger$ (Fig. S1-S4). Density functional theory (DFT) calculations at the B3LYP/6$31 \mathrm{G}(\mathrm{d}, \mathrm{p})$ level were used to calculate the highest occupied molecular orbital (HOMO) and the lowest unoccupied molecular orbital (LUMO) energies of the donor cores (IDTT, ST, 4T and ST) and the terminal groups (BA, IC and DFIC), in addition to the dipole moments of the terminal groups. As shown in Fig. 1a, 4T, ST and 6T central cores have a higher HOMO level than that of IDTT, indicating their better electron-donating ability, which favors the NIR absorbing capability of FREAs. Regarding the terminal groups, BA's LUMO level is much higher than that of the IC group, which is the highest among the four terminal groups, indicating its weakest electron-withdrawing ability. Compared to the IC group, fluorinated IC groups possess a deeper LUMO level which agrees with the reported values. The different electronwithdrawing ability of terminal groups deriving from their different electronegativity can be compared by calculating their regional dipole moments shown in Fig. 1b. The dipole moments of $4.166,4.439,4.884$ and $5.420 \mathrm{D}$ were observed for the Se-BA, T-BA, T-IC and T-DFIC segments, respectively. It is clear that the dipole moment of T-BA is significantly different from that of T-DFIC, which may induce unbalanced charge transport in two segment based binary acceptors (vide infra).

\subsection{Electrochemical and optical properties}

The absorption and energy level of the third component acceptor are widely considered to be crucial for efficient ternary OSCs. In order to eliminate the interference of these two factors and study the effect of electronegativity in ternary OSCs, six ternary blends with appropriate energy levels and complementary absorption are constructed (Fig. 2). The electrochemical properties of all these materials were investigated by cyclic voltammetry (CV) in the same condition (Fig. 2a and Table S1, Fig. S5, ESI $\dagger$ ). From the onset of oxidation potentials in the $\mathrm{CV}$, the HOMO energy level of PBDT2TC was determined to be $-5.37 \mathrm{eV}$, while its LUMO level was difficult to obtain from the $\mathrm{CV}$ measurement due to the unreliable reduction data. Thus, we used the optical bandgap and HOMO level to estimate PBDT-2TC's LUMO level to be $-3.41 \mathrm{eV}$. The HOMO/LUMO energy levels of the new acceptors 4TBA and STBA were estimated to be $-5.51 \mathrm{eV} /-3.70 \mathrm{eV}$ and $-5.45 \mathrm{eV} /-3.60 \mathrm{eV}$, 
(a)

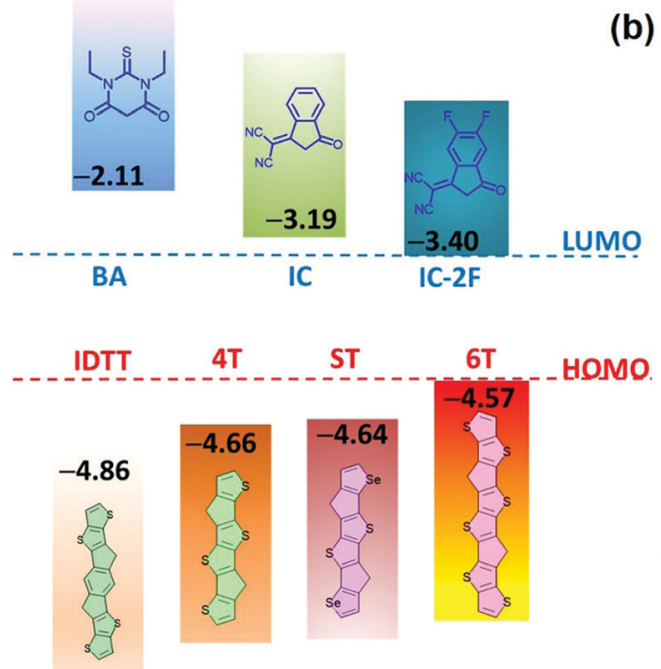

(b)
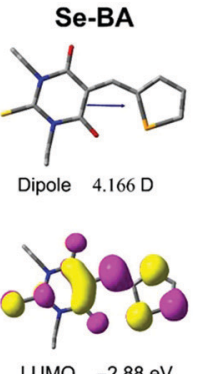

LUMO $-2.88 \mathrm{eV}$

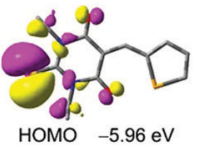

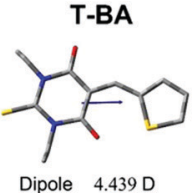
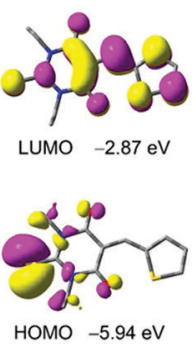

T-IC

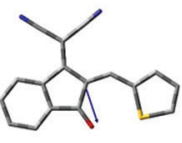

Dipole $4.884 \mathrm{D}$
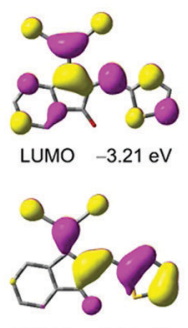

HОMO $-6.42 \mathrm{eV}$
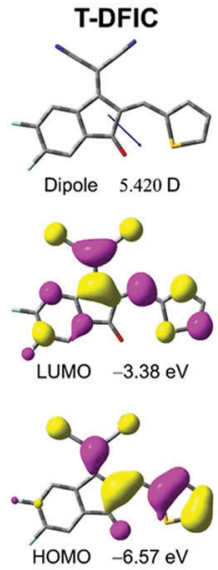

Fig. 1 (a) Energy levels of cores and end groups calculated by DFT. (b) Dipole moments for the regional parts (Se-BA, T-BA, T-IC, T-FIC and T-DFIC) in the asymmetric molecules.

(a)
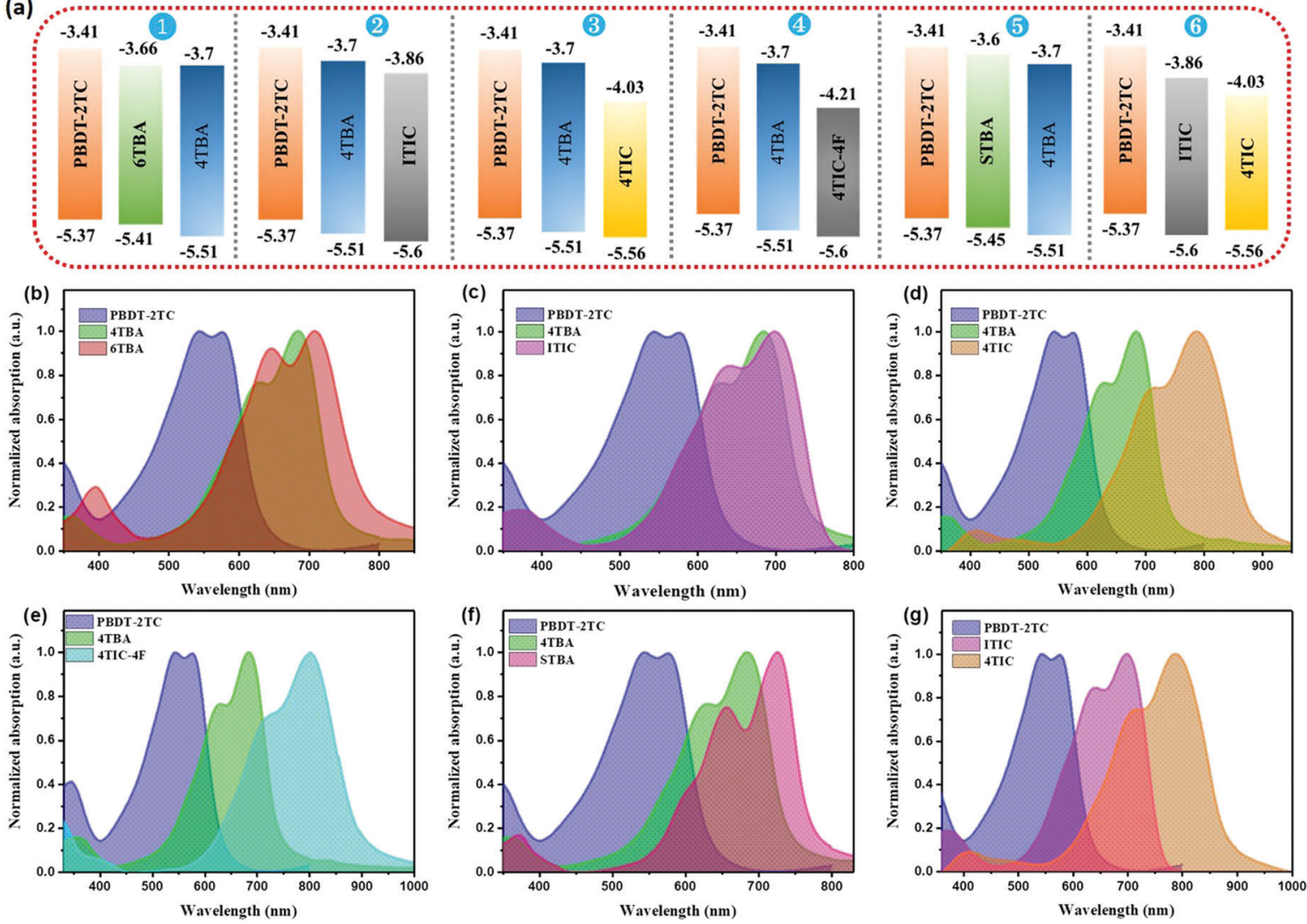

Fig. 2 (a) Energy diagrams of six ternary systems' materials. (b-g) Normalized UV-vis absorption spectra for the materials of six ternary blends.

respectively. Similarly, the HOMO/LUMO energy levels of 6TBA, ITIC, 4TIC and 4TIC-4F were estimated to be $-5.41 \mathrm{eV} /-3.66 \mathrm{eV}$, $-5.60 \mathrm{eV} /-3.86 \mathrm{eV},-5.56 \mathrm{eV} /-4.03 \mathrm{eV}$ and $-5.60 \mathrm{eV} /-4.21 \mathrm{eV}$, respectively.

Evidently, the first five ternary blends all exhibit cascade energy levels, which is believed to be beneficial for charge transfer.
Notably, the sixth ternary blend shows multiple electron charge transfer, and nonradiative Förster resonance energy transfer (FRET) between ITIC and 4TIC, which may improve the device performance. The normalized UV-vis absorption profiles of PBDT2TC and FREAs in the film state and six ternary blends are shown in Fig. $2 \mathrm{~b}-\mathrm{g}$ and the absorption spectra of the new acceptors $4 \mathrm{TBA}$ 
and STBA in dichloromethane solution are presented in Fig. S6, ESI. $\dagger$ From the UV-vis spectra, 4TBA exhibits strong absorption from 550 to $730 \mathrm{~nm}$, which is expected to complement the absorption of PBDT-2TC. The 4TBA film shows an absorption edge at $746 \mathrm{~nm}$ corresponding to an optical bandgap of $1.66 \mathrm{eV}$. Compared to 4TBA, STBA exhibits redshifted absorption $(780 \mathrm{~nm})$ and reduced bandgap (1.59 eV), which is ascribed to the more electron-rich ST unit and stronger intramolecular charge transfer of STBA. 6TBA, ITIC, 4TIC and 4TIC-4F, which constitute the third component, all show redshifted absorption compared to the host acceptor 4TBA, which can potentially benefit the absorption of the ternary blends. As a result, the six ternary blends all display complementary absorption, which is beneficial for lightharvesting. Thus, the potential interference from the factors (appropriate energy levels and complementary absorption) in the six selected ternary blends has been eliminated, which is helpful to study the effect of dipole moment in ternary OSCs.

\subsection{Photovoltaic device performance}

Ternary OSCs were fabricated in a conventional device configuration of ITO/PEDOT:PSS/PBDT-2TC:acceptor1:acceptor2/C60-bis/ $\mathrm{Ag}$, where $\mathrm{C}_{60}$-bis is the bis-fulleropyrrolidium iodide salt. ${ }^{50}$ The molecular structure of C60-bis and the device structure are shown in Fig. 3a and b, respectively. The acceptor1 in this work is 4TBA and the binary BHJ device of PBDT-2TC:4TBA gave the best performance under the optimal condition of donor/acceptor weight ratio $1: 1$ in 1,2-dichlorobenzene (DCB) solution with thermal annealing at $120{ }^{\circ} \mathrm{C}$ for $10 \mathrm{~min}$ at $2000 \mathrm{rpm}$. The details of device optimization are provided in Tables S2 and S3, ESI. $\dagger$ Then, the weight ratio was kept at 1:1 (polymer : total acceptor) for all ternary blends with only the acceptors' relative weight ratio being tuned. We first optimized the ternary blend PBDT2TC:4TBA:6TBA. As shown in Table 1 and Fig. 3c, the binary device based on PBDT-2TC:4TBA exhibited a high $V_{\text {oc }}$ of $0.962 \mathrm{~V}$ and a FF of 0.71 , delivering a high PCE of $10.52 \%$. On varying the amount of 6TBA in ternary blends, the $V_{\mathrm{oc}}$ of the related devices increased while $J_{\text {sc }}$ and FF increased initially but then decreased gradually. When the ratio of 4TBA: 6TBA was $9: 1$, the highest PCE of $11.73 \%$ was obtained with a $V_{\text {oc }}$ of $0.968 \mathrm{~V}$, a $J_{\mathrm{sc}}$ of $16.6 \mathrm{~mA} \mathrm{~cm}^{-2}$, and a $\mathrm{FF}$ of 0.73 .

Next, the weight ratio was kept at 1:0.9:0.1 for other five ternary blends and all the other conditions of device fabrication were kept consistent to perform a fair comparison of important device parameters. Then, a third component ITIC which has a similar absorption range replaced 6TBA and the ternary blend PBDT-2TC:4TBA:ITIC was constructed. Different to the previous ternary system, the $V_{\mathrm{oc}}, J_{\mathrm{sc}}$ and $\mathrm{FF}$ of the ternary blend device all dropped, leading to a decreased PCE of $8.73 \%$. The decreased performance is due to the large difference between the donor core of ITIC and 4TBA. Based on this, 4TIC which has the same donor core as 4TBA and the same terminal group as ITIC was used to build another ternary OSC. Similarly, we found that although the 4TIC absorption broadened and even redshifted to $900 \mathrm{~nm}$, the device performance of PBDB-2TC:4TBA:4TIC also significantly decreased (PCE decreased from $10.52 \%$ to $6.35 \%$ ). More notably, the decrease is more pronounced (PCE decreased from $10.52 \%$ to $3.57 \%$ ) when fluorine atoms are introduced at the end of 4TIC in the PBDT-2TC:4TBA:4TIC-4F ternary system. Thus, we speculate that the large difference in the dipole moment between the two acceptors' terminal groups could affect the performance of ternary OSCs.

To confirm the effect of dipole moment in ternary OSCs and exclude the interference of the donor core, the performance of another two ternary blends of PBDT-2TC:4TBA:STBA and PBDT2TC:ITIC:4TIC with the same terminal but different donor cores was investigated. In spite of the huge difference in the donor core, the PCE of the PBDT-2TC:4TBA:STBA blend decreased little compared to that of the binary blend (PCE decreased from
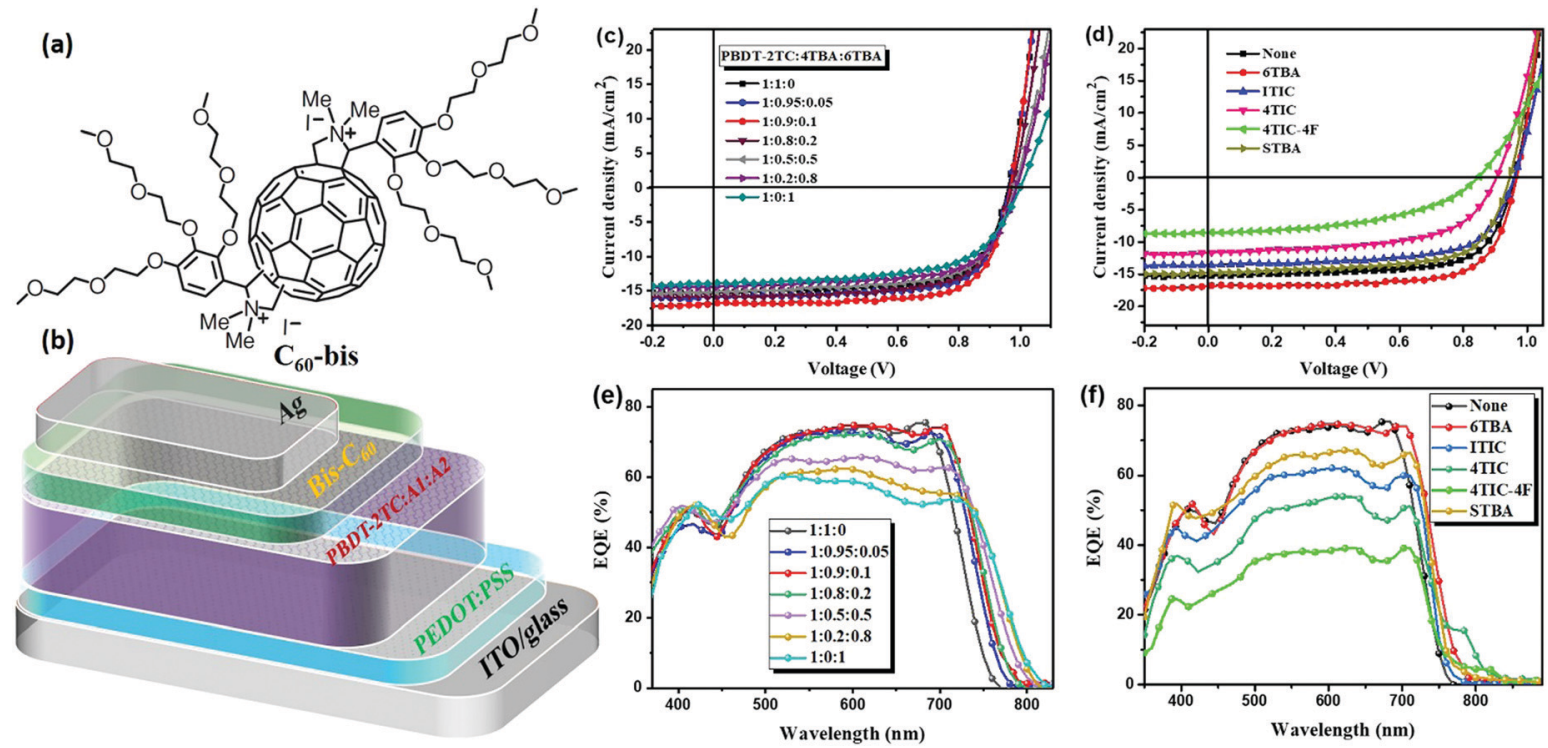

Fig. 3 (a) Molecular structure of $\mathrm{C}_{60}$-bis. (b) Device architecture. (c) J-V curves of PBDT-2TC:4TBA:6TBA based OSCs with different acceptor contents. (d) $J-V$ curves of multiple ternary system based OSCS (PBDT-2TC: 4TBA: acceptor2 = 1:0.9:0.1). (e and f) EQE spectra of the respective ternary devices. 
Table 1 Photovoltaic parameters of OSCs based on ternary blends

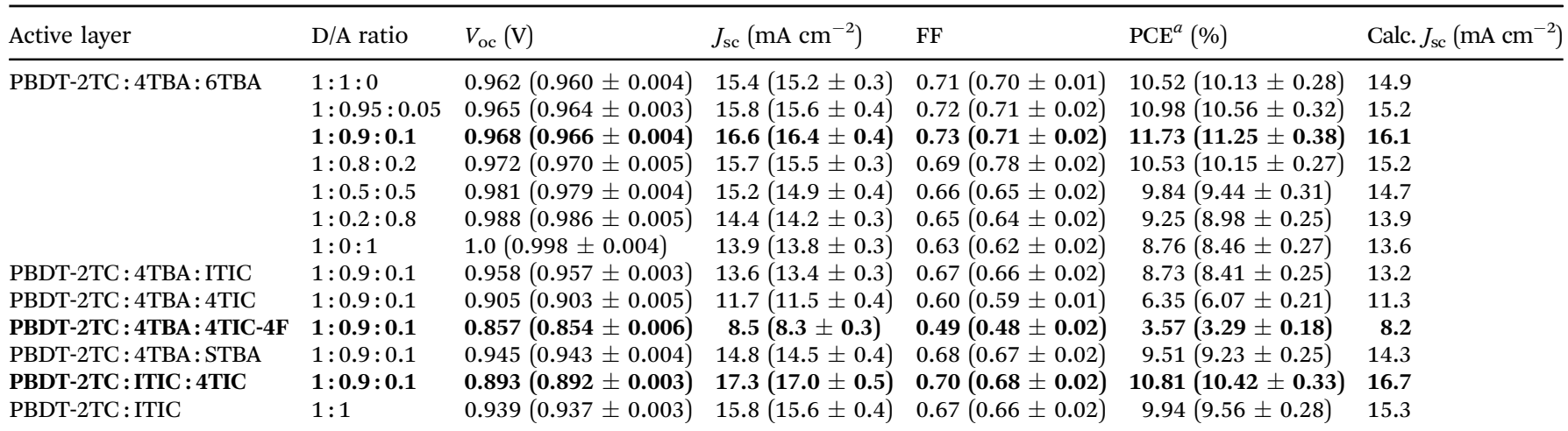

${ }^{a}$ Average values obtained from ten devices are shown in parentheses.

(a)
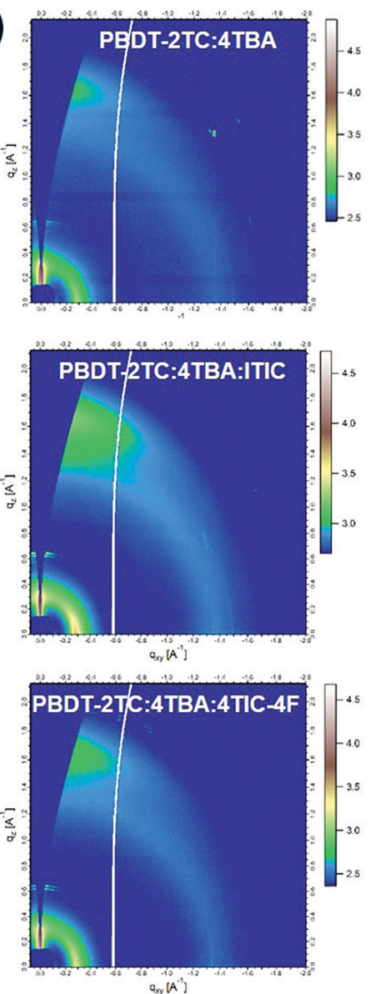
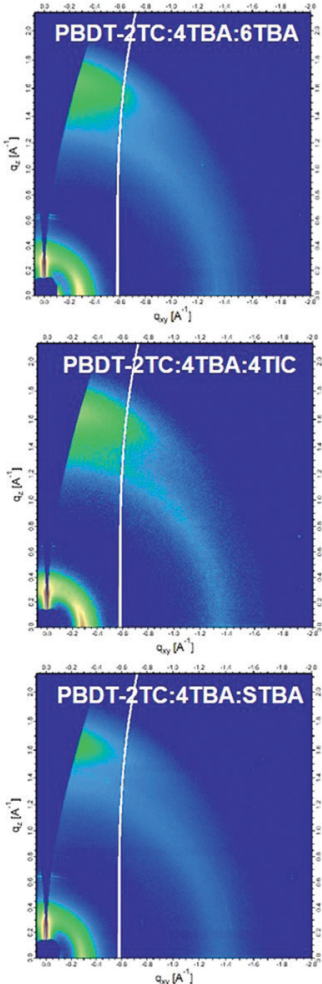

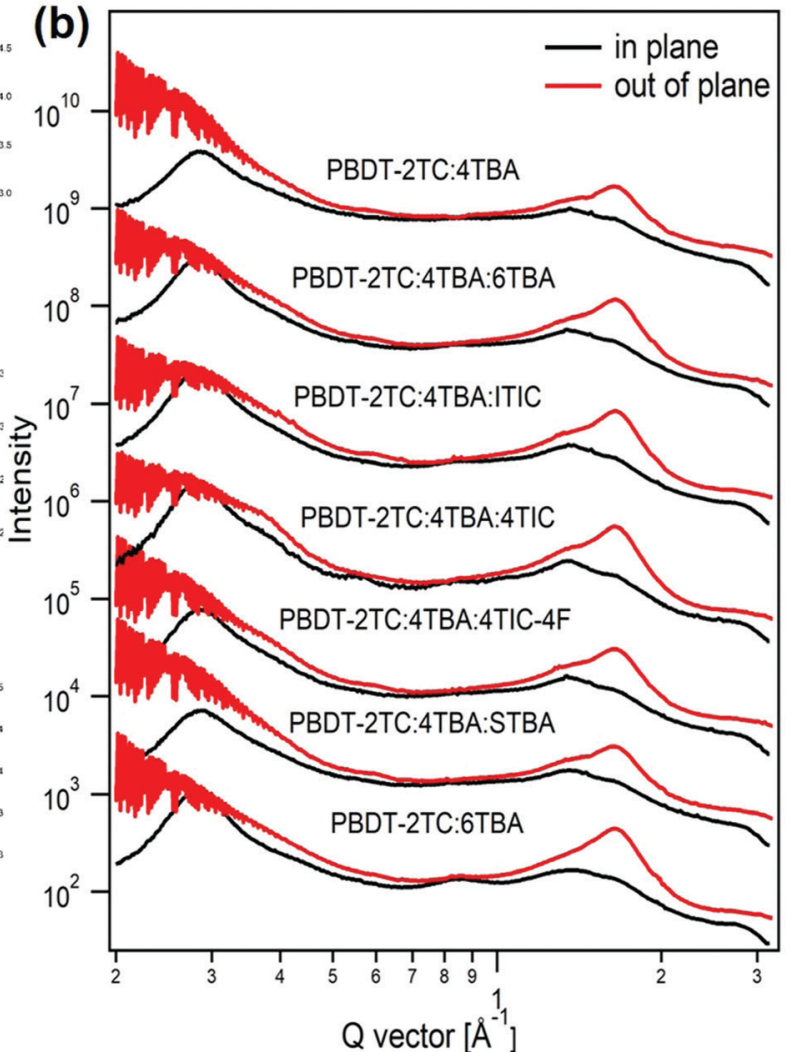

Fig. 4 (a) 2D GIWAXS patterns for PBDT-2TC:4TBA, PBDT-2TC:4TBA:6TBA, PBDT-2TC:4TBA:ITIC, PBDT-2TC:4TBA:4TIC, PBDT-2TC:4TBA:4TIC-4F, and PBDT-2TC:4TBA:STBA. (b) In-plane (black line) and out-of-plane (red line) line-cut profiles of GIWAXS results.

$10.52 \%$ to $9.51 \%)$. The device of PBDT-2TC:ITIC:4TIC worked well and its PCE improved from $9.94 \%$ to $10.81 \%$, as shown in Table 1 and Fig. S7, ESI. $\dagger$ Therefore, from the results of the above six ternary OSCs, we can see that the terminals' dipole moment of the combined acceptors has a more significant impact on device performance, especially for the key parameters $\mathrm{FF}$ and $J_{\mathrm{sc}}$. The changes in $J_{\mathrm{sc}}$ were confirmed by external quantum efficiency (EQE) measurements, as shown in Fig. 3e and f. Fig. 3e displays the EQE curves of PBDT2TC:4TBA:6TBA ternary devices with different acceptor ratios.
The PBDT-2TC:4TBA based binary device showed a high EQE response in the wavelength region of $350-750 \mathrm{~nm}$. After the addition of $10 \%$ 6TBA, the EQE broadened to $780 \mathrm{~nm}$, which is consistent with the broadened absorption of 6TBA. Fig. 3f shows the EQE curves of the multiple ternary OSCs. It is clear that the intensity of $\mathrm{EQE}$ response significantly decreased after the addition of ITIC, 4TIC and especially 4TIC-4F. From the integration of the EQE curves, the calculated $J_{\mathrm{sc}}$ was obtained, which is within a reasonable difference to the $J_{\mathrm{sc}}$ values obtained from the corresponding $J-V$ curves (Table 1 ). 


\subsection{Morphology analysis}

The morphology of the $\mathrm{BHJ}$ active layer plays a significant role in affecting the device performance. In order to differentiate if the performance of the above ternary OSCs is really affected by the dipole moment of the acceptor's terminal group or is influenced by BHJ morphology, we have carried out the grazing incidence wide-angle scattering (GIWAXS) measurements ${ }^{51}$ on the samples studied above. GIWAXS can provide the information of molecular packing and crystalline orientation of these ternary BHJs. The GIWAXS 2D patterns and the corresponding line-cut profiles are shown in Fig. 4 and Fig. S8, ESI. $\dagger$ It is obvious that all the binary and ternary films exhibit a face-on favorable orientation, as proven by the prominent (010) $\pi-\pi$ stacking peaks in the out-of-plane direction. ${ }^{52,53}$ For all ternary films, the addition of the third component didn't cause any significant change in morphology compared to that of binary PBDT-2TC:4TBA and PBDT-2TC:6TBA films. In addition, all the ternary films exhibit similar (100) lamellar stacking peaks at $q \approx 0.28 \AA^{-1}$ in the in-plane direction and similar (010) $\pi-\pi$ stacking peaks at $q \approx 1.65 \AA^{-1}$ in the out-of-plane direction, suggesting that all the ternary films exhibit similar molecular packing and crystalline orientation. However, the device performances of these multiple ternary OSCs are quite different from each other. Therefore, the differences in performance among these ternary OSCs are not really caused by the morphology of the $\mathrm{BHJ}$ active layers.

\subsection{The role of dipole moment and its impact on charge transfer}

To confirm the effect of dipole moment on ternary OSCs and study the working mechanism, steady state photoluminescence (PL) and time-resolved PL (TRPL) were performed. As shown in Fig. 5a, we first studied the effect of the blend ratio on the charge transfer properties of the PBDT-2TC:4TBA:6TBA ternary blend. The peak emission of the pure PBDT-2TC film is located at $642 \mathrm{~nm}$. For the blend films, the PL emission of PBDT-2TC is almost completely quenched and when the ternary blend (PBDT-2TC : 4TBA : 6TBA) weight ratio is $1: 0.9: 0.1$, the PL peak is most significantly quenched. Other ternary blends (shown in Fig. 5b) also exhibited similar results, demonstrating that the photoexcited electrons could be efficiently transferred from the polymer donor to the acceptors. This result agrees well with previous literature which reported that when FREAs' end groups approach the electron-enriched backbone of the polymer donor, ${ }^{45}$ the strong dipole effect of the end groups may facilitate the charge transfer process.

Among these ternary blends, PBDT-2TC:4TBA:6TBA displayed the fastest exciton dissociation revealed by its shortest exciton lifetime (1.56 ns) which was fitted from the TPRL spectra (Fig. 5c). This favorable charge separation efficiency could also contribute to the higher $J_{\mathrm{sc}}$ and photovoltaic performance. In comparison, the ternary blend PBDT-2TC:4TBA:4TIC-4F showed the longest exciton lifetime of $3.87 \mathrm{~ns}$, suggesting the slowest exciton dissociation in the active layer. It is very interesting that the speed of exciton dissociation differs among the ternary blends while the difference in overall charge separation efficiency is similar (revealed by PL quenching efficiency, Fig. 5b). This indicates that the dipole moment may have little effect on the processes of charge separation and transfer between donor and acceptor. It may have a stronger impact on the subsequent charge transport between the acceptors as the electrons are transported through the acceptor materials.

To investigate the terminal's dipole moment on ternary OSCs' charge transport, the electron and hole mobility of multiple ternary blend films was determined using the space-chargelimited-current method $^{54}$ with single-carrier device architectures of ITO/ZnO/active layer/ $\mathrm{C}_{60}$-bis/Ag and ITO/PEDOT:PSS/active
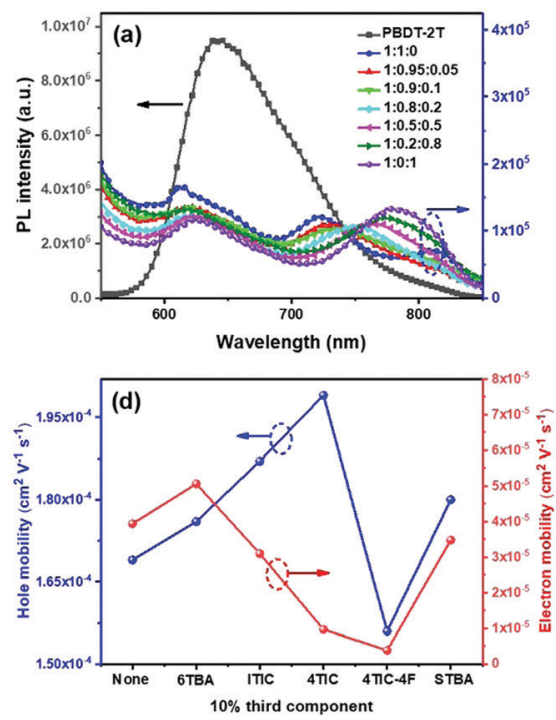
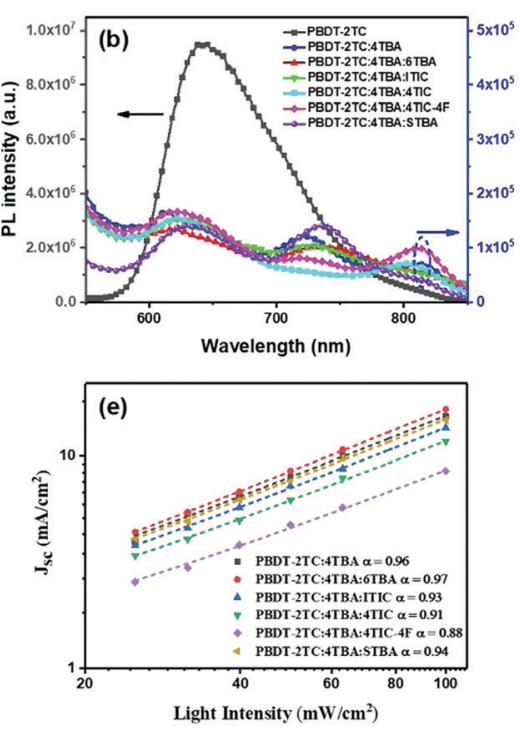

Fig. 5 (a) PL spectra of the pristine polymer film and active blend films with different weight ratios of 4TBA:6TBA. (b) PL spectra of the pristine polymer film and PBDT-2TC:4TBA blended films with or without a different third acceptor component (10 wt\%). (c) TRPL spectra of PBDT-2TC:4TBA blended films with or without a different third acceptor component (10 wt\%). (d) Hole mobility and electron mobility dependence on the $10 \mathrm{wt} \%$ third component. Light intensity dependence of (e) $J_{s c}$ and (f) $V_{o c}$ of the blended films with or without a different third acceptor component. 
Table 2 The parameters of hole and electron mobility

\begin{tabular}{lllr}
\hline & \multicolumn{3}{c}{$u_{\mathrm{h}}$} \\
Active layer & {$\left[10^{-4} \mathrm{~cm}^{2} \mathrm{~V}^{-1} \mathrm{~s}^{-1}\right]\left[10^{-5} \mathrm{~cm}^{2} \mathrm{~V}^{-1} \mathrm{~s}^{-1}\right]$} & $u_{\mathrm{h}} / u_{\mathrm{e}}$ \\
\hline PBDT-2TC:4TBA & 1.69 & 3.94 & 4.23 \\
PBDT-2TC:4TBA:6TBA & 1.76 & 5.06 & 3.48 \\
PBDT-2TC:4TBA:ITIC & 1.87 & 3.10 & 6.03 \\
PBDT-2TC:4TBA:4TIC & 1.99 & 0.97 & 20.52 \\
PBDT-2TC:4TBA:4TIC-4F & 1.56 & 0.39 & 40 \\
PBDT-2TC:4TBA:STBA & 1.80 & 3.48 & 5.17
\end{tabular}

$u_{\mathrm{h}}$ : hole mobility; $u_{\mathrm{e}}:$ electron mobility. The balance of hole and electron mobility $\left(u_{\mathrm{h}} / u_{\mathrm{e}}\right)$.

layer $/ \mathrm{MoO}_{3} / \mathrm{Ag}$, respectively. As shown in Fig. 5d, Table 2 and Fig. S9, ESI, $\dagger$ both the binary and multiple ternary devices exhibited a high hole mobility $\left(u_{\mathrm{h}}\right)$ with a small variation (1.56$1.99 \times 10^{-4} \mathrm{~cm}^{2} \mathrm{~V}^{-1} \mathrm{~s}^{-1}$ ). Compared to the binary blend, the hole mobility for the ternary blends PBDT-2TC:4TBA:6TBA, PBDT2TC:4TBA:ITIC and PBDT-2TC:4TBA:4TIC was improved which may be due to the increased crystallinity of the BHJ morphology. Nevertheless, the electron mobility $\left(u_{\mathrm{e}}\right)$ is strongly dependent on the difference of terminal's electronegativity between two FREAs in ternary blends. Compared to the $u_{\mathrm{e}}$ for the binary blend (3.94 $\times 10^{-5} \mathrm{~cm}^{2} \mathrm{~V}^{-1} \mathrm{~s}^{-1}$ ), the $u_{\mathrm{e}}$ for the ternary blend PBDT-2TC:4TBA:4TIC-4F decreased dramatically to $3.89 \times$ $10^{-6} \mathrm{~cm}^{2} \mathrm{~V}^{-1} \mathrm{~s}^{-1}$, leading to significantly unbalanced charge mobility $\left(u_{\mathrm{h}} / u_{\mathrm{e}}=40.1\right)$, which is detrimental to charge transport and collection, and thus resulting in low $J_{\mathrm{sc}}$ and $\mathrm{FF}$ values. For the ternary blends with two acceptors based on the same terminals, the charge transport balance of $u_{\mathrm{h}} / u_{\mathrm{e}}$ is 3.48 and 5.17 for PBDT2TC:4TBA:6TBA and PBDT-2TC:4TBA:STBA, respectively. Meanwhile, for the ternary blends with two acceptors based on different terminals, the charge transport becomes more and more unbalanced $\left(u_{\mathrm{h}} / u_{\mathrm{e}}=6.03\right.$ for PBDT-2TC:4TBA:ITIC, $u_{\mathrm{h}} / u_{\mathrm{e}}=$ 20.54 for PBDT-2TC:4TBA:4TIC), which could result in increased charge recombination (vide infra). These results demonstrate that the terminals' dipole moment of acceptors could affect the charge transport and play an important role in ternary OSCs.

\subsection{Recombination analysis}

To confirm that the unbalanced charge transport could result in increased charge recombination, we have studied the dependence of $J_{\mathrm{sc}}$ and $V_{\mathrm{oc}}$ on light intensity ( $I$ ), as shown in Fig. 5e and f. In general, the relationship between $J_{\mathrm{sc}}$ and $I$ follows the power-law formula of $J_{\mathrm{sc}} \propto I^{\alpha}$, where the slope of the curve $(\alpha)$ reflects the bimolecular recombination degree; if $\alpha$ is closer to unity, it suggests the weak bimolecular recombination in active layers. ${ }^{55,56}$ Fig. 5e depicts the plots of $\log J_{\mathrm{sc}}$ versus $\log I$; the $\alpha$ values are $0.96,0.97$ and 0.94 for the PBDT-2TC:4TBA binary device, the PBDT-2TC: 4TBA:6TBA ternary device and the PBDT-2TC:4TBA:STBA ternary device, respectively, implying less charge recombination loss during charge extraction in the PBDT-2TC:4TBA:6TBA devices. Clearly, in the ternary systems based on two acceptors with terminals of different dipole moment, the $\alpha$ values decreased to 0.93 and 0.91 for PBDT-2TC:4TBA:ITIC and PBDT-2TC:4TBA: 4TIC, respectively, and significantly decreased to 0.88 for PBDT2TC:4TBA:4TIC-4F, suggesting more charge recombination in these ternary blends. The recombination mechanisms in these devices can be further investigated according to the relationship of $V_{\mathrm{oc}}$ dependence on light intensity, as shown in Fig. $5 \mathrm{f}$. The charge recombination process in blend films can be defined by the formula $V_{\text {oc }} \propto n\left(k_{\mathrm{B}} T / q\right) \ln I$, where $k_{\mathrm{B}}, T$ and $q$ are the Boltzmann constant, absolute temperature and elementary charge, respectively. ${ }^{57}$ A stronger dependence of $V_{\mathrm{oc}}$ on light intensity with a slope greater than $k T / q$ is observed when trapassisted recombination is involved. In our cases, the dependence of $V_{\mathrm{oc}}$ on the light intensity for ternary devices based on two acceptors with terminals of different electronegativity was recorded with a much higher slope than that based on two acceptors with terminals of same dipole moment. The result also indicates less trap-assisted recombination in these ternary devices based on combined acceptors with more compatible dipole moment. Therefore, the study of charge recombination mechanisms proved that the unbalanced charge transport which is derived from the difference in the dipole moment of terminals between two acceptors could result in increased charge recombination, thus significantly decreasing $J_{\mathrm{sc}}$ and FF in these ternary devices.

\subsection{The mechanism of hypothesis}

From the investigations of photophysical and photovoltaic properties, and the $\mathrm{BHJ}$ morphology, we have confirmed the influence of the dipole moment of acceptor's terminal groups in ternary OSCs. In two FREA based ternary OSCs, two acceptors with the same terminals invariably work well than acceptors with different terminals. Based on these results, the compatible dipole moment seems to dominate the performance of the ternary blends, and thus a schematic diagram of such a working principle is proposed and presented in Fig. 6. If the terminals in two FREAs in the ternary blends have a huge difference in electron-withdrawing ability, it would result in unbalanced charge transport, leading to increased charge combination in ternary OSCs. Consequently, the performance of the devices will significantly decrease in spite of adding only a small amount of the third component. In contrast, if the two FREAs in the ternary blends possess different fused-rings but the same terminals, the blends exhibit more balanced charge transport and less charge recombination. Under the circumstances, the two FREAs with high compatibility result in better device performance.

To further verify the hypothesis, additional DFT calculation analysis was performed. It is well known that the strong $\pi-\pi$ stacking between conjugated organic molecules can facilitate charge transport, thereby improving the performance of OSCs, and the $\pi-\pi$ stacking effect can be calculated from the dimer configurations. ${ }^{58}$ The plots of electron density $\rho(r)$ multiplied by the sign of the second Hessian eigenvalue $\lambda_{2}$ versus reduced density gradient (RDG) $\left(\mathrm{RDG}=\frac{1}{2\left(3 \pi^{2}\right)^{1 / 3}} \frac{|\nabla \rho|}{\rho^{4 / 3}}\right)$ for the dimers of six systems (4TBA:6TBA, 4TBA:ITIC, 4TBA:4TIC, 4TBA:4TIC-4F, 4TBA:STBA and 4TIC:ITIC) were created to understand the interaction nature for different stacking modes in Fig. S9 (ESI $\dagger$ ) and Fig. 7. The blue, green and red areas in Fig. 7 stand for hydrogen bonds, van der Waals interactions and steric repulsion, respectively. ${ }^{59}$ The lowest 
(a)

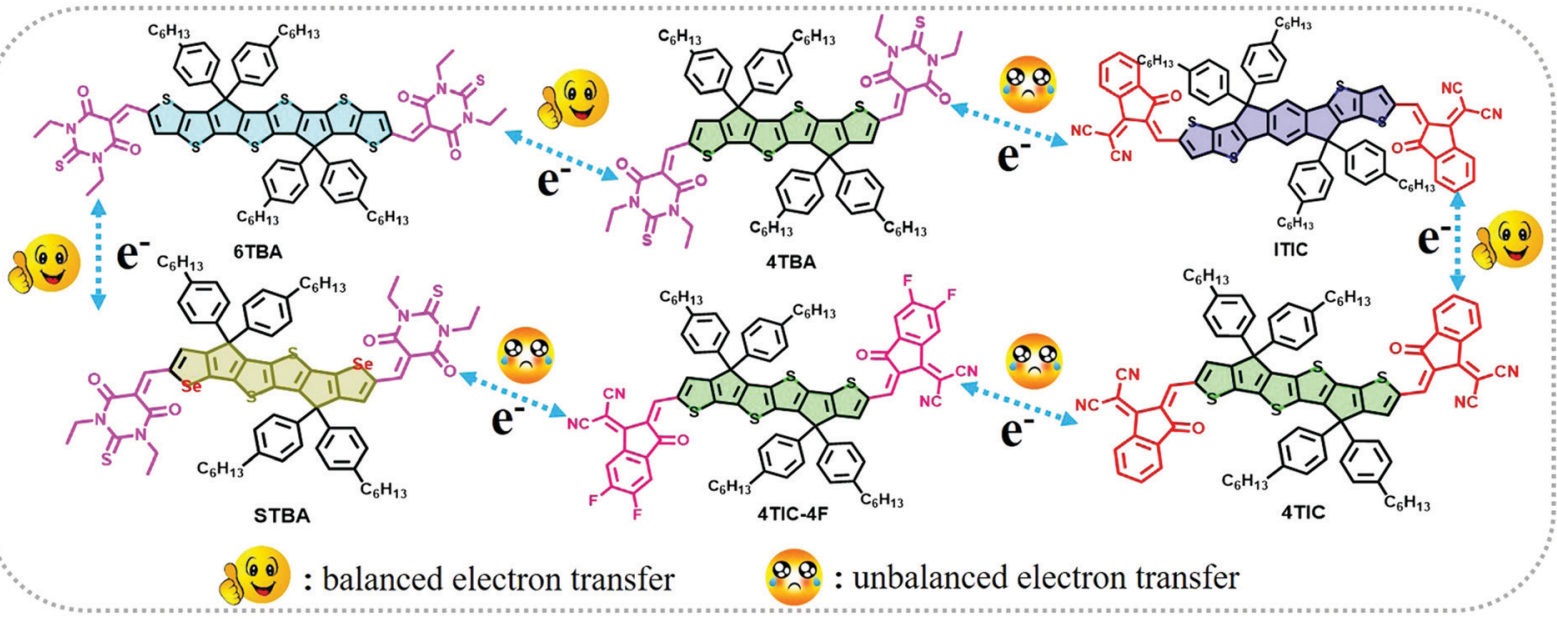

(b)
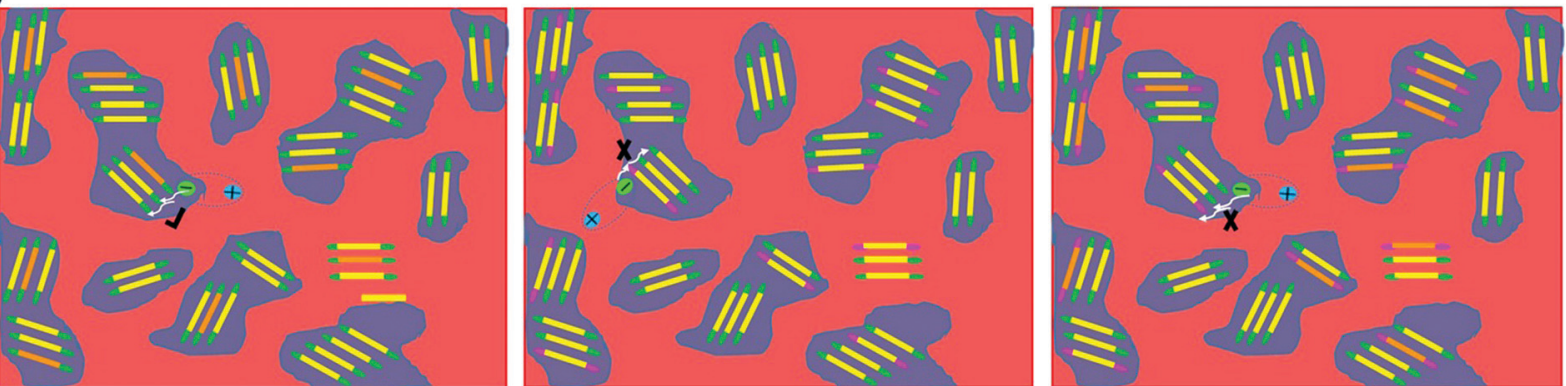

Polymer phase

FREA phase

electron

+ hole $\equiv$ FREAs

Fig. 6 (a) Hypothetical mechanism diagram of electron transport in ternary blends reflected by molecular structures. (b) The possible charge transport pathway existing in three different types of ternary systems; the yellow and orange segments in FREAs represent the donor cores, the green and pink segments represent terminals and different colors represent different donor cores or terminals.

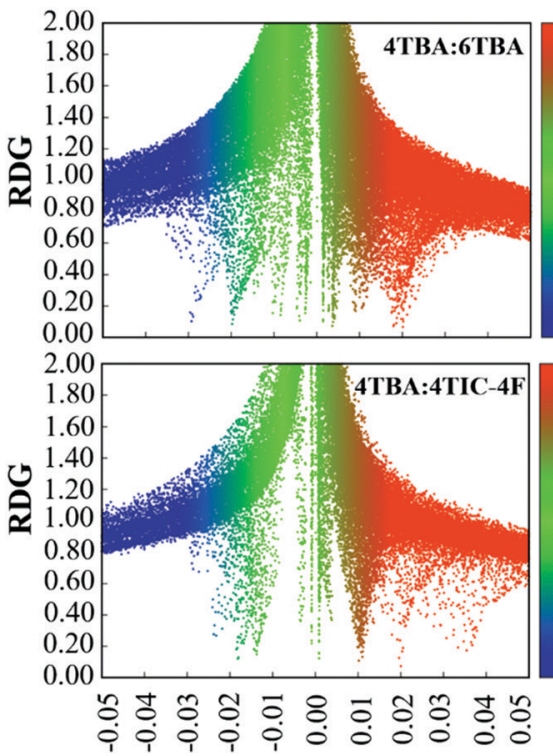

$\operatorname{Sign}\left(\lambda_{2}\right) \rho($ a.u. $)$
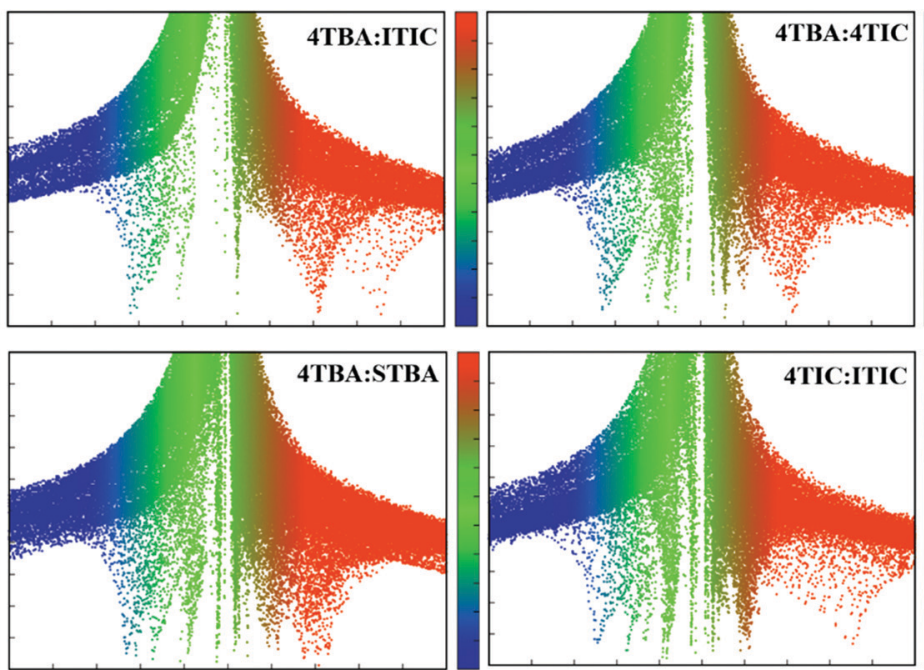

0.020

0.015

0.005

0.000

$-0.005$

$-0.010$

$-0.015$

$-0.020$

$-0.025$

$-0.030$

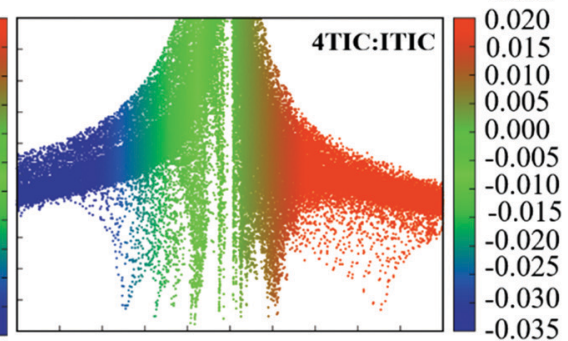

$-0.035$

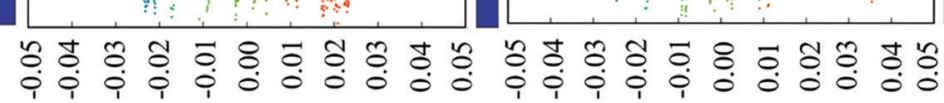

$\operatorname{Sign}\left(\lambda_{2}\right) \rho$ (a.u.)

Fig. 7 The plots of the production of electron density $\rho(r)$ and the sign of the second Hessian eigenvalue $\lambda_{2}$ versus RDG for six combinations of 4TBA:6TBA, 4TBA:ITIC, 4TBA:4TIC, 4TBA:4TIC-4F, 4TBA:STBA and 4TIC:ITIC.

energy dimer structures were obtained by the Molclus program ${ }^{60}$ and Gaussian. Additionally, RDG analysis of the terminal stacking regions between the acceptor molecules was performed using the Multiwfn software. ${ }^{61}$ The $\pi-\pi$ stacking interactions with a value of 
$\operatorname{sign}\left(\lambda_{2}\right) \rho$ around zero between the acceptor molecules' terminals are clearly manifested by the gradient isosurfaces ( $\mathrm{RDG}=0.5$ a.u.). From Fig. S9 (ESI $\dagger$ ) and Fig. 7, it can be seen that the dimers (4TBA:6TBA, 4TBA:STBA, 4TIC:ITIC) with the same dipole moment terminals have better $\pi-\pi$ interactions with more gradient isosurfaces. However, the gradient isosurface area between the 4TBA and $4 \mathrm{TIC}-4 \mathrm{~F}$ terminals is the smallest, indicating that the dimers have the worst $\pi-\pi$ interactions. The worst $\pi-\pi$ stacking interaction in the 4TBA:4TIC-4F blend may be detrimental to charge transfer and transport, which may explain why the PBDT-2TC: 4TBA:4TIC-4F based device has significant unbalanced charge mobility. These results indicate that the terminals' dipole moment of FREAs plays an important role in ternary OSCs, which is consistent with our hypothesis.

\section{Conclusions}

In summary, a new important parameter of dipole moment is established in designing and evaluating the performance of ternary OSCs based on one conjugated polymer and two FREAs. Six ternary blends with complementary absorption and cascade energy levels were systematically constructed. From the results of studying these six ternary OSCs, we found that if there is a huge difference in two acceptors' terminal dipole moment, the electron mobility will be significantly decreased to result in unbalanced charge transport and more charge recombination, thus inducing significant attenuated device performance. As a result, the PBDT-2TC:4TBA:6TBA blend shows the best performance while the PBDT-2TC:4TBA:4TIC-4F blend exhibits the worst performance even when a small amount of the third component 4TIC-4F is added. This is the first time that a comprehensive study has been conducted to understand the role of dipole moment in affecting ternary OSCs. This method can be used as a rough guideline to screen ternary systems for improving the device performance.

\section{Author contributions}

X. L. and X. S. conceived the idea and designed the experiment with the help of L. C., L. Z., K. G. and F. L.; X. L. performed the device fabrication and data analysis; Z. Y. and Y. C. carried out the DFT simulation; Y. A. and Q. X. made the polymer PBDT2CT which was not purchased; H. Z. and W. M. performed the GIWAXS characterization and data analysis; P. Z., Y. G. and X. F. helped in the analysis of the electrochemical measurements; X. L. wrote the original draft; All authors discussed the results and commented on the manuscript; Y. W. C. supervised the project.

\section{Conflicts of interest}

There are no conflicts to declare.

\section{Acknowledgements}

X. L. acknowledges the support from "the Fundamental Research Funds for the Central Universities” (2232019D3-04), Shanghai
Sailing Program (19YF1401000), the National Natural Science Foundation of China (NSFC) (51973032, and 21905043), and the Initial Research Funds for Young Teachers of Donghua University. A. J. acknowledges the financial support from the Office of Naval Research (N00014-17-1-2201). Y. C. thanks for support from the National Natural Science Foundation of China (NSFC) (51673091 and 51833004). X-ray data were acquired at beamlines 7.3.3 and 11.0.1.2 at Lawrence Berkeley National Laboratory, which is supported by the DOE, Office of Science, and Office of Basic Energy Sciences.

\section{Notes and references}

1 Y. Lin, J. Wang, Z. G. Zhang, H. Bai, Y. F. Li, D. B. Zhu and X. Zhan, An Electron Acceptor Challenging Fullerenes for Efficient Polymer Solar Cells, Adv. Mater., 2015, 27, 1170.

2 C. Yan, S. Barlow, Z. Wang, H. Yan, A. K.-Y. Jen, S. R. Marder and X. Zhan, Non-fullerene acceptors for organic solar cells, Nat. Rev. Mater., 2018, 3, 18003.

3 S. Chen, H. Cho, J. Lee, Y. Yang, Z. Zhang, Y. Li and C. Yang, Modulating the Molecular Packing and Nanophase Blending via a Random Terpolymerization Strategy toward 11\% Efficiency Nonfullerene Polymer Solar Cells, Adv. Energy Mater., 2017, 1701125.

4 M. Jeong, S. Chen, S. Lee, Z. Wang, Y. Yang, Z. Zhang, C. Zhang, M. Xiao, Y. Li and C. Yang, Feasible D1-A-D2-A Random Copolymers for Simultaneous High-Performance Fullerene and Nonfullerene Solar Cells, Adv. Energy Mater., 2017, 1702166.

5 Y. Wang, Z. Peng, S. Xiao, J. Yang, H. Zhou, L. Huang, L. Sun, Y. Zhou, L. Tan and Y. Chen, Highly stable Al-doped ZnO by ligand-free synthesis as general thickness-insensitive interlayers for organic solar cells, Sci. China: Chem., 2018, 61, 127. $6 \mathrm{X} . \mathrm{Xu}, \mathrm{Z} . \mathrm{Bi}, \mathrm{W} . \mathrm{Ma}, \mathrm{Z}$. Wang, W. C. H. Choy, W. Wu, G. Zhang, Y. Li and Q. Peng, Highly efficient ternary-blend polymer solar cells enabled by a nonfullerene acceptor and two polymer donors with a broad composition tolerance, Adv. Mater., 2017, 29, 1704271.

7 Y. Zhang, L. Shi and Y. Chen, Overview and Outlook of Random Copolymerization Strategy for Designing Polymer Solar Cells, Acta Polym. Sin., 2019, 50, 13.

8 S. Li, L. Ye, W. Zhao, H. Yan, B. Yang, D. Liu, W. Li, H. Ade and $\mathrm{J}$. Hou, A wide band gap polymer with a deep highest occupied molecular orbital level enables $14.2 \%$ efficiency in polymer solar cells, J. Am. Chem. Soc., 2018, 140, 7159.

9 D. Liu, J. Wang, C. Gu, Y. Li, X. Bao and R. Yang, Stirring Up Acceptor Phase and Controlling Morphology via Choosing Appropriate Rigid Aryl Rings as Lever Arms in SymmetryBreaking Benzodithiophene for High-Performance Fullerene and Fullerene-Free Polymer Solar Cell, Adv. Mater., 2018, 1705870.

10 Y. Yang, Z. Zhang, H. Bin, S. Chen, L. Xue, C. Yang and Y. Li, Side-chain isomerization on an n-type organic semiconductor ITIC acceptor makes $11.77 \%$ high efficiency polymer solar cells, J. Am. Chem. Soc., 2016, 138, 15011. 
11 F. Zhao, S. Dai, Y. Wu, Q. Zhang, J. Wang, L. Jiang, Q. Ling, Z. Wei, W. Ma, W. You, C. Wang and X. Zhan, Singlejunction binary-blend nonfullerene polymer solar cells with 12.1\% efficiency, Adv. Mater., 2017, 29, 1700144.

12 H. Yao, Y. Cui, R. Yu, B. Gao, H. Zhang and J. Hou, Design, synthesis, and photovoltaic characterization of a small molecular acceptor with an ultra-narrow band gap, Angew. Chem., Int. Ed., 2017, 56, 3045.

13 C. Huang, X. Liao, K. Gao, L. Zuo, F. Lin, X. Shi, C.-Z. Li, F. Liu, Y. Chen, H. Chen and A. K.-Y. Jen, Highly efficient organic solar cells based on $\mathrm{S}, \mathrm{N}$-heteroacene non-fullerene acceptors, Chem. Mater., 2018, 30, 5429.

14 J. Sun, X. Ma, Z. Zhang, J. Yu, J. Zhou, X. Yin, L. Yang, R. Geng, R. Zhu, F. Zhang and W. Tang, Dithieno[3,2-b:2', $\left.3^{\prime}-d\right]$ pyrrol Fused Nonfullerene Acceptors Enabling Over 13\% Efficiency for Organic Solar Cells, Adv. Mater., 2018, 1707150.

15 Z. Yao, X. Liao, K. Gao, F. Lin, X. Xu, X. Shi, L. Zuo, F. Liu, Y. Chen and A. K.-Y. Jen, Dithienopicenocarbazole-based acceptors for efficient organic solar cells with optoelectronic response over $1000 \mathrm{~nm}$ and an extremely low energy loss, J. Am. Chem. Soc., 2018, 140, 2054.

16 Z. Xiao, X. Jia and L. Ding, Ternary organic solar cells offer 14\% power conversion efficiency, Sci. Bull., 2017, 62, 1562.

17 J. Yuan, Y. Zhang, L. Zhou, G. Zhang, H. Yip, T. Lau, X. Lu, C. Zhu, H. Peng and P. Johnson, et al., Single-junction organic solar cell with over $15 \%$ efficiency using fused-ring acceptor with electron-deficient core, Joule, 2019, 3, 1140.

18 X. Shi, L. Zuo, S. Jo, K. Gao, F. Lin, F. Liu and A. K.-Y. Jen, Design of a highly crystalline low-band gap fused-ring electron acceptor for high-efficiency solar cells with low energy loss, Chem. Mater., 2017, 29, 8369.

19 J. Zhang, H. S. Tan, X. Guo, A. Facchetti and H. Yan, Material insights and challenges for non-fullerene organic solar cells based on small molecular acceptors, Nat. Energy, 2018, 3, 720.

20 Y. Cui, H. Yao, B. Gao, Y. Qin, S. Zhang, B. Yang, C. He, B. Xu and J. Hou, Fine-tuned photoactive and interconnection layers for achieving over $13 \%$ efficiency in a fullerene-free tandem organic solar cell, J. Am. Chem. Soc., 2017, 139, 7302.

21 W. Li, A. Furlan, K. H. Hendriks, M. M. Wienk and R. A. J. Janssen, Efficient tandem and triple-junction polymer solar cells, J. Am. Chem. Soc., 2013, 135, 5529.

22 F. Chen, J. Xu, Z. Liu, M. Chen, R. Xia, Y. Yang, T. K. Lau, Y. Zhang, X. Lu, H. L. Yip, A. K. Jen, H. Chen and C. Li, NearInfrared Electron Acceptors with Fluorinated Regioisomeric Backbone for Highly Efficient Polymer Solar Cells, Adv. Mater., 2018, 30, 1803769.

23 Y. Li, J. D. Lin, X. Liu, Y. Qu, F. P. Wu, F. Liu, Z. Q. Jiang and S. R. Forrest, Near-Infrared Ternary Tandem Solar Cells, Adv. Mater., 2018, 30, 1804416.

24 L. Zuo, X. Shi, S. B. Jo, Y. Liu, F. Lin and A. K. Jen, Tackling energy loss for high-efficiency organic solar cells with integrated multiple strategies, Adv. Mater., 2018, 30, 1706816.

25 L. Meng, Y. Zhang, X. Wan, C. Li, X. Zhang, Y. Wang, X. Ke, Z. Xiao, L. Ding, R. Xia, H.-L. Yip, Y. Cao and Y. Chen,
Organic and solution-processed tandem solar cells with 17.3\% efficiency, Science, 2018, 361, 1094.

26 Y. Yang, W. Chen, L. T. Dou, W. H. Chang, H. S. Duan, B. Bob, G. Li and Y. Yang, High-performance multiple-donor bulk heterojunction solar cells, Nat. Photonics, 2015, 9, 190. 27 N. Gasparini, L. Lucera, M. Salvador, M. Prosa, G. D. Spyropoulos, P. Kubis, H.-J. Egelhaaf, C. J. Brabec and T. Ameri, High-performance ternary organic solar cells with thick active layer exceeding 11\% efficiency, Energy Environ. Sci., 2017, 10, 885.

28 L. Y. Lu, M. A. Kelly, W. You and L. P. Yu, Status and prospects for ternary organic photovoltaics, Nat. Photonics, 2015, 9, 491.

29 X. Liao, J. Wang, S. Chen, L. Chen and Y. Chen, Diketopyrrolopyrrole-based conjugated polymers as additives to optimize morphology for polymer solar cells, Chin. J. Polym. Sci., 2016, 34, 491.

30 D. Baran, T. Kirchartz, S. Wheeler, S. Dimitrov, M. Abdelsamie, J. Gorman, R. S. Ashraf, S. Holliday, A. Wadsworth, N. Gasparini, P. Kaienburg, H. Yan, A. Amassian, C. J. Brabec, J. R. Durrant and I. McCulloch, Reduced voltage losses yield 10\% efficient fullerene free organ-ic solar cells with $>1 \mathrm{~V}$ open circuit voltages, Energy Environ. Sci., 2016, 9, 3783.

31 W. Li, L. Yang, J. R. Tumbleston, L. Yan, H. Ade and W. You, Controlling Molecular Weight of a High Efficiency DonorAcceptor Conjugated Polymer and Understanding Its Signifi-cant Impact on Photovoltaic Properties, Adv. Mater., 2014, 26, 4456.

32 B. A. Collins, Z. Li, J. R. Tumbleston, E. Gann, C. R. McNeill and $\mathrm{H}$. Ade, Absolute Measurement of Domain Composition and Nanoscale Size Distribution Explains Performance in PTB7:PC ${ }_{71}$ BM Solar Cells, Adv. Energy Mater., 2013, 3, 65.

33 H. Zhou, L. Yang and W. You, Rational design of high performance conjugated polymers for organic solar cells, Macromolecules, 2012, 45, 607.

34 K. Jiang, G. Zhang, G. Yang, J. Zhang, Z. Li, T. Ma, H. Hu, W. Ma, H. Ade and H. Yan, Multiple Cases of Efficient Nonfullerene Ternary Organic Solar Cells Enabled by an Effective Morphology Control Method, Adv. Energy Mater., 2017, 1701370.

35 M. Zhang, W. Gao, F. J. Zhang, Y. Mi, W. B. Wang, Q. S. An, J. Wang, X. L. Ma, J. L. Miao, Z. H. Hu, X. F. Liu, J. Zhang and C. L. Yang, Efficient ternary non-fullerene polymer solar cells with PCE of $11.92 \%$ and FF of 76.5, Energy Environ. Sci., 2018, 11, 841.

36 Z. H. Luo, H. J. Bin, T. Liu, Z. G. Zhang, Y. K. Yang, C. Zhong, B. B. Qiu, G. H. Li, W. Gao, D. J. Xie, K. L. Wu, Y. M. Sun, F. Liu, Y. F. Li and C. L. Yang, Fine-Tuning of Molecular Packing and Energy Level through Methyl Substitution Enabling Excellent Small Molecule Acceptors for Nonfullerene Polymer Solar Cells with Efficiency up to 12.54\%, Adv. Mater., 2018, 30, 1706124.

37 Q. An, J. Zhang, W. Gao, F. Qi, M. Zhang, X. Ma, C. Yang, L. Huo and F. Zhang, Efficient Ternary Organic Solar Cells with Two Compatible Non-Fullerene Materials as One Alloyed Acceptor, Small, 2018, 14, 1802983.

38 J. Lee, S. J. Ko, M. Seifrid, H. Lee, C. McDowell, B. R. Luginbuhl, A. Karki, K. Cho, T. Q. Nguyen and G. C. Bazan, 
Design of Nonfullerene Acceptors with Near-Infrared Light Absorption Capabilities, Adv. Energy Mater., 2018, 8, 1801209.

39 X. L. Ma, W. Gao, J. S. Yu, Q. S. An, M. Zhang, Z. H. Hu, J. X. Wang, W. H. Tang, C. L. Yang and F. J. Zhang, Ternary nonfullerene polymer solar cells with efficiency $>13.7 \%$ by integrating the advantages of the materials and two binary cells, Energy Environ. Sci., 2018, 11, 2134.

40 H. Zhang, H. F. Yao, J. X. Hou, J. Zhu, J. Q. Zhang, W. N. Li, R. N. Yu, B. W. Gao, S. Q. Zhang and J. H. Hou, Over 14\% efficiency in organic solar cells enabled by chlorinated nonfullerene small-molecule acceptors, Adv. Mater., 2018, 30, 1800613.

41 Q. An, J. Wang, W. Gao, X. Ma, Z. Hu, J. Gao, C. Xu, M. Hao, X. Zhang, C. Yang and F. Zhang, Alloy-like ternary polymer solar cells with over $17.2 \%$ efficiency, Sci. Bull., 2020, DOI: 10.1016/j.scib.2020.01.012.

42 T. Yan, W. Song, J. Huang, R. Peng, L. Huang and Z. Ge, 16.67\% Rigid and $14.06 \%$ Flexible Organic Solar Cells Enabled by Ternary Heterojunction Strategy, Adv. Mater., 2019, 1902210.

43 M. Pan, T. Lau, Y. Tang, Y. Wu, T. Liu, K. Li, M. M. Chen, X. Lu, W. Ma and C. Zhan, 16.7\%-efficiency ternary blended organic photovoltaic cells with PCBM as the acceptor additive to increase the open-circuit voltage and phase purity, J. Mater. Chem. A, 2019, 7, 20713.

44 L. Zhan, S. Li, T. Lau, Y. Cui, X. Lu, M. Shi, C. Li, H. Li, J. Hou and $\mathrm{H}$. Chen, Over $17 \%$ efficiency ternary organic solar cells enabled by two non-fullerene acceptors working in alloy-like model, Energy Environ. Sci., 2020, 13, 635.

45 Z. Zheng, O. Awartani, B. Gautam, D. Liu, Y. Qin, W. Li, A. Bataller, K. Gundogdu, H. Ade and J. Hou, Efficient charge transfer and fine-tuned energy level alignment in a THF-processed fullerene-free organic solar cell with $11.3 \%$ efficiency, Adv. Mater., 2017, 29, 1604241.

46 M. Li, Y. Zhou, J. Zhang, J. Song and Z. Bo, Tuning the dipole moments of nonfullerene acceptors with an asymmetric terminal strategy for highly efficient organic solar cells, J. Mater. Chem. A, 2019, 7, 8889.

47 Y. An, X. Liao, L. Chen, J. Yin, Q. Ai, Q. Xie, B. Huang, F. Liu, A. K.-Y. Jen and Y. Chen, Nonhalogen Solvent-Processed Asymmetric Wide-Bandgap Polymers for Nonfullerene Organic Solar Cells with Over 10\% Efficiency, Adv. Funct. Mater., 2018, 28, 1706517.

48 L. Zuo, X. Shi, S. Jo, Y. Liu, F. Lin and A. K.-Y. Jen, Tackling energy loss for high-efficiency organic solar cells with integrated multiple strategies, Adv. Mater., 2018, 30, 1706816.
49 X. Shi, X. Liao, K. Gao, L. Zuo, J. Chen, J. Zhao, F. Liu, Y. Chen and A. K.-Y. Jen, An Electron Acceptor with Broad Visible-NIR Absorption and Unique Solid State Packing for As-Cast High Performance Bi-nary Organic Solar Cells, Adv. Funct. Mater., 2018, 28, 1802324.

50 C.-Z. Li, C.-C. Chueh, H.-L. Yip, K. M. O’Malley, W.-C. Chen and A. K.-Y. Jen, Effective interfacial layer to enhance efficiency of polymer solar cells via solution-processed fullerene-surfactants, J. Mater. Chem., 2012, 22, 8574.

51 A. Hexemer, W. Bras, J. Glossinger, E. Schaible, E. Gann, R. Kirian, A. MacDowell, M. Church, B. Rude and H. Padmore, A SAXS/WAXS/GISAXS beamline with multilayer mono-chromator, J. Phys.: Conf. Ser., 2010, 247, 012007.

52 L. Zhang, B. Lin, B. Hu, X. Xu and W. Ma, Blade-Cast Nonfullerene Organic Solar Cells in Air with Excellent Morphology, Efficiency, and Stability, Adv. Mater., 2018, 30, 1800343.

53 L. Zhang, X. Xu, B. Lin, H. Zhao, T. Li, J. Xin, Z. Bi, G. Qiu, S. Guo, K. Zhou, X. Zhan and W. Ma, Achieving Balanced Crystallinity of Donor and Acceptor by Combining BladeCoating and Ternary Strategies in Organic Solar Cells, Adv. Mater., 2018, 30, 1805041.

54 V. Mihailetchi, J. Wildeman and P. Blom, Space-charge limited photocurrent, Phys. Rev. Lett., 2005, 94, 126602.

55 A. K. K. Kyaw, D. H. Wang, V. Gupta, W. L. Leong, L. Ke, G. C. Bazan and A. J. Heeger, Intensity dependence of current-voltage characteristics and recombination in highefficiency solution-processed small-molecule solar cells, ACS Nano, 2013, 7, 4569.

56 R. Ingo, P. Jürgen, D. Vladimir, L. Laurence, V. Dirk and C. H. Jan, Effect of Temperature and Illumination on the Electrical Char-acteristics of Polymer-Fullerene BulkHeterojunction Solar Cells, Adv. Funct. Mater., 2004, 14, 38.

57 L. J. A. Koster, V. D. Mihailetchi, R. Ramaker and P. W. M. Blom, Light intensity dependence of open-circuit voltage of polymer: fullerene solar cells, Appl. Phys. Lett., 2005, 86, 1235.

58 G. Han, Y. Yi and Z. Shuai, From molecular packing structures to electronic processes: theoretical simulations for organic solar cells, Adv. Energy Mater., 2018, 8, 1702743.

59 E. R. Johnson, S. Keinan, P. Mori-Sánchez, J. ContrerasGarcia, A. J. Cohen and W. Yang, Revealing noncovalent interactions, J. Am. Chem. Soc., 2010, 132, 6498.

60 T. Lu, Molclus Program, Version 1.9, http://www.keinsci. com/research/molclus.html.

61 T. Lu and F. Chen, Multiwfn: a multifunctional wavefunction analyser, J. Comput. Chem., 2012, 33, 580. 